\title{
Le bassin du Rhin à l'amont de Bâle et l'influence des lacs sur le régime du fleuve
}

\section{The catchment area of the Rhine above Basle and the influence of the lakes on the river's regime}

\author{
PAR E. WALSER
}

GHEF DU SERVICE HYDROGRAPHIQUe AU SERVICE FÉDÉRAL DES EAUX a BERNE.

\begin{abstract}
Les données caractéristiques du bassin du Rhin à l'amont de Bále sont résumées et comparées avec celles du bassin total du Rhin et, partiellement avec celles du bassin du Rhin $\dot{a}$ l'aval de Bâle. Ceci, d'abord en ce qui concerne la configuration et la topographie; ensuite en ce qui concerne les débits et leurs variations dans le temps. Les glaciers et les lacs sont caracteristiques pour le bassin supérieur, ils manquent dans le bassin inférieur.

Le coefficient d'écoulement est indiqué et il est montré qu'il varie avec l'altitude. Le régime des débits à Bâle, dit régime alpin, est caractérisé et les facteurs conditionnant ce régime sont discutés.

Après une brève discussion des crues hivernales, le régime purement alpin des émissaires des glaciers est mis en relief.

Ensuite, l'influence des lacs sur le régime des débits à Bâle est discutée, influence non seulement saisonnière, mais aussi régulatrice sur les crues et sur le transport de matériaux solides. Par d'importants travanx de correction en Suisse, la partie des apports compensés par des lacs a été, au courant du XVIII ${ }^{\circ}$ et du $X I X^{e}$ siècles, sensiblement augmentée.
\end{abstract}

The characteristics of the Rhine upstream of Basle are summarized and compared with the charncteristics of the complete Rhine Basin and are partially compared with the characteristics of the Rhine Basin downstream of Bale. Configuration and topography are dealt with first of all, then flow and how it varies with time. Glaciers and lakes characterize the upper basin but are not encountered in the lower basin.

The runoff coefficient is given and it is shown that it varies with altitude. The flow regimen at Bale is alpine and is discussed together with the factors that condition it.

After a short discrussion of winter floods, the purely alpine nature of the regimens of the glacial tributaries is brought out.

The next point to be discussed is the influence of the Iaties on the flow regimen at Basle. This effect is not only seasonal but it also regulates fooding and the transport of solid materials. Large scale training work during the eighteenth and nineteenth centuries appreciably increased the flow-smoothing effect of the lakes.

\section{I. - LE BASSIN DU RHIN A L'AMONT DE BALE PAR RAPPORT A LA TOTALITE DU BASSIN DU RHIN}

Si nous suivons le cours du Rhin sur une carte de l'Europe Centrale, nous voyons clairement que Bâle est un des points les plus mar. quants (fig. 1). On remarque en effet que le fleuve après avoir suivi tout d'abord les directions nord-est et nord vers le lac de Constance et s'être dirigé ensuite vers l'ouest pour traver- ser les monts du Jura, prend une orientation définitive vers le nord à Bâle, où il entre dans la basse plaine du Haut-Rhin. D'autres facteurs encore - que nous exposerons tout à l'heure - justifient l'attention particulière que nous accordons au bassin du Rhin à l'amont de Bâle dans notre exposé des régions traversées 


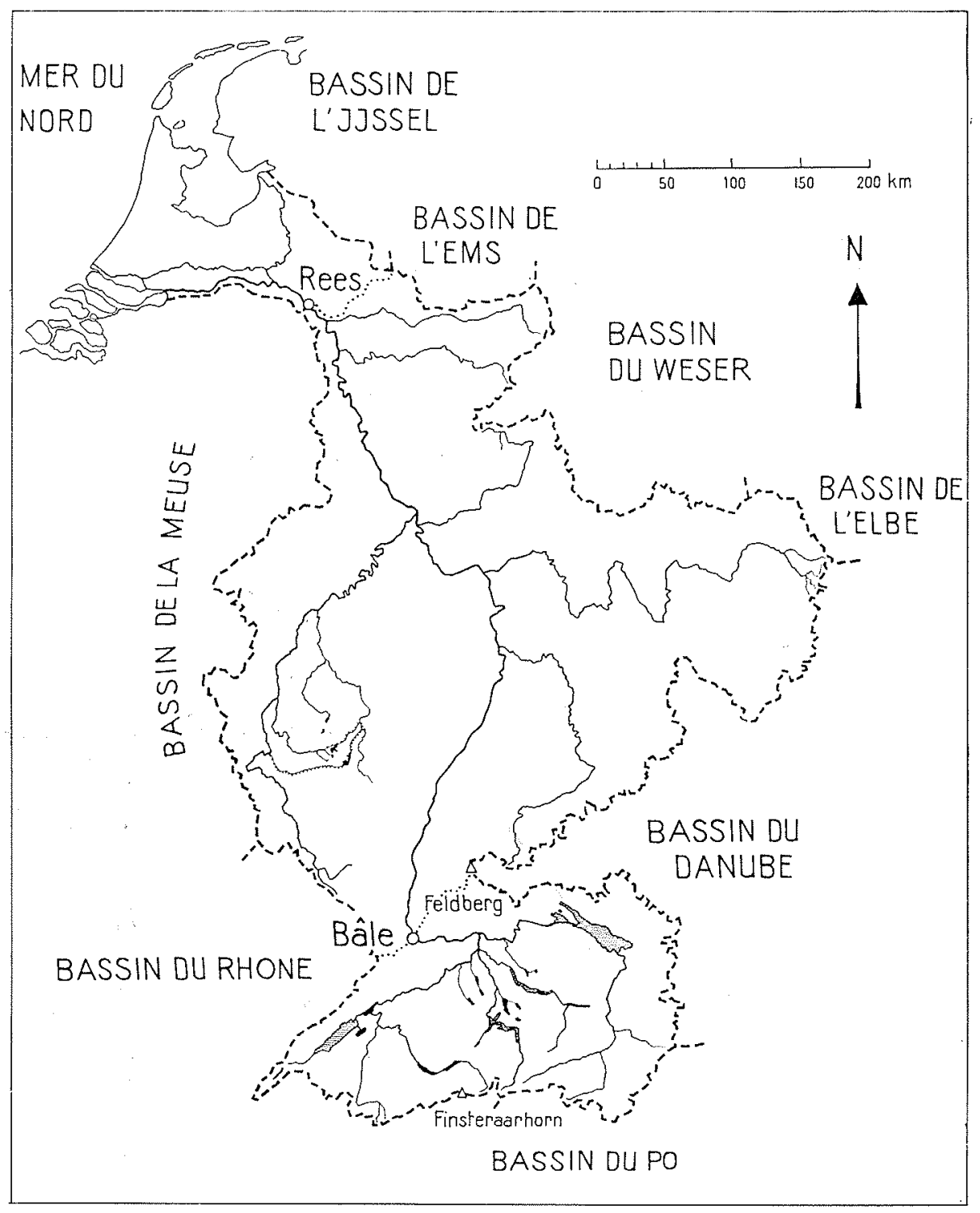

Frg. 1

Bassin du Rhin depuis ses sources jusqu'à

la mer du Nord. par ce fleuve, et à cette fin, nous comparerons quelques aspects de cette partie de son cours avec le bassin total, c'est-à-dire son bassin de réception jusqu'à Rees, près de la frontière germano-néerlandaise.

Les lignes de partage des eaux des principaux bassins, indiquées sur la figure 1, font bien apparaitre comment, près de Bâle, les bassins du Rhône et du. Danube se rapprochent à quelque $90 \mathrm{~km}$, de sorte qu'ils arrivent presque à séparer la partie amont du bassin du Rhin de sa partie aval. Alors que la surface totale du bassin de réception du Rhin - des sources jusqu'à Rees - est donnée comme étant de $159683 \mathrm{~km}^{2}$ [4], la partie du bassin en amont de Bâle (jusques et y compris la Wiese) est de $36492 \mathrm{~km}^{2}$ [1], c'est-à-dire $22,9 \%$ de la super- ficie totale. Le caractère spécial de ce bassin partiel devient d'autant plus frappant si l'on considère le relief de la région. Le point culminant $d u$ bassin global est le Finsteraarhorn à $4.274 \mathrm{~m}$ d'altitude, alor's que celui de la partie du bassin en aval de Bâle n'est que de $1493 \mathrm{~m}$ (Feldberg). Le lac Toma, considéré comme la source du Rhin Antérieur (Vorder-Rhein) est situé à une altitude de $2341 \mathrm{~m}$ (fig. 3), le niveau du lac de Constance à $396 \mathrm{~m}$; à Bâle, le plan d'eau est encore à $244 \mathrm{~m}$. La répartition des surfaces du bassin de réception en fonction de leur altitude est représentée par la couche hypsographique [2], dont nous disposons pour la région à l'amont de Bâle, et qui est reproduite sur la figure 2. Nous l'avons également complétée pour le bassin total, sur la base de mesures au plani- 


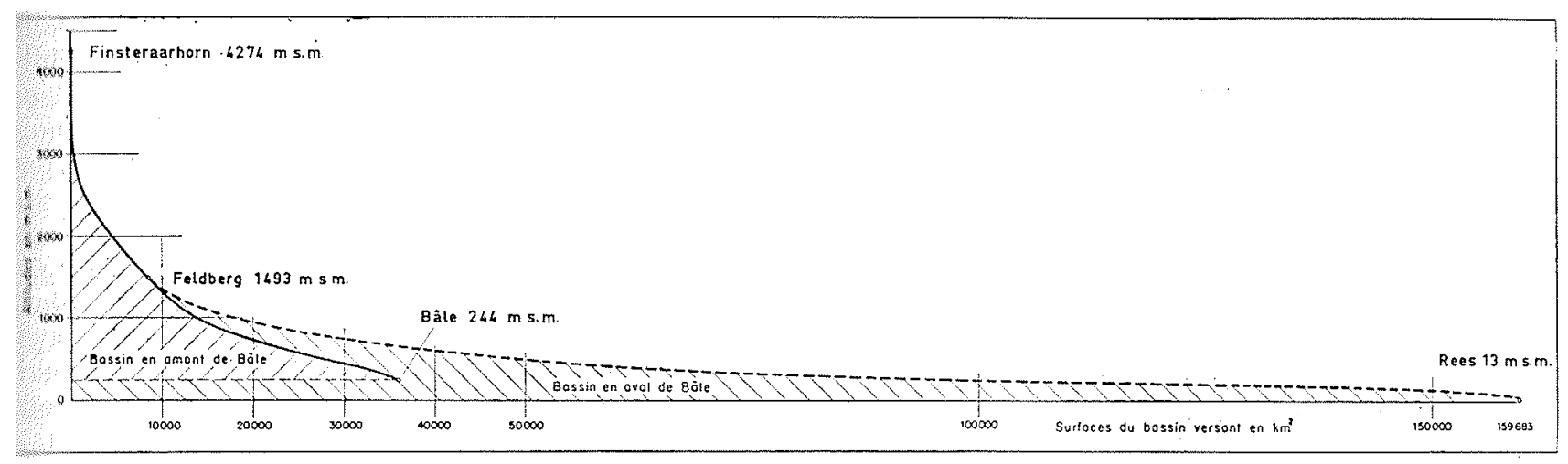

FIG. 2

Courbe hypsométrique du bassin du Rhin en amont de Bâle et jusqu'à Rees. Un point de la coube hypsométrique foumit la surface partielle du bassin (abscisse) située au-dessus de l'altitude (ordonnée) du. point considéré.

motre d'une carte physique de l'Europe Cenrale; bien que plutôt sommaire, ce procédé est néanmoins adéquat pour le but recherché. On y voit clairement les différences considérables cutre les zones de hauteurs dans lesquelles se situent de grandes parties des deux bassins de réception partiels, d'une part celui à l'amont de Bâle, et d'autre part celui à l'aval de cette ville. Les influences de ces conditions sur le régime d'écoulement seront particulièrement mises en

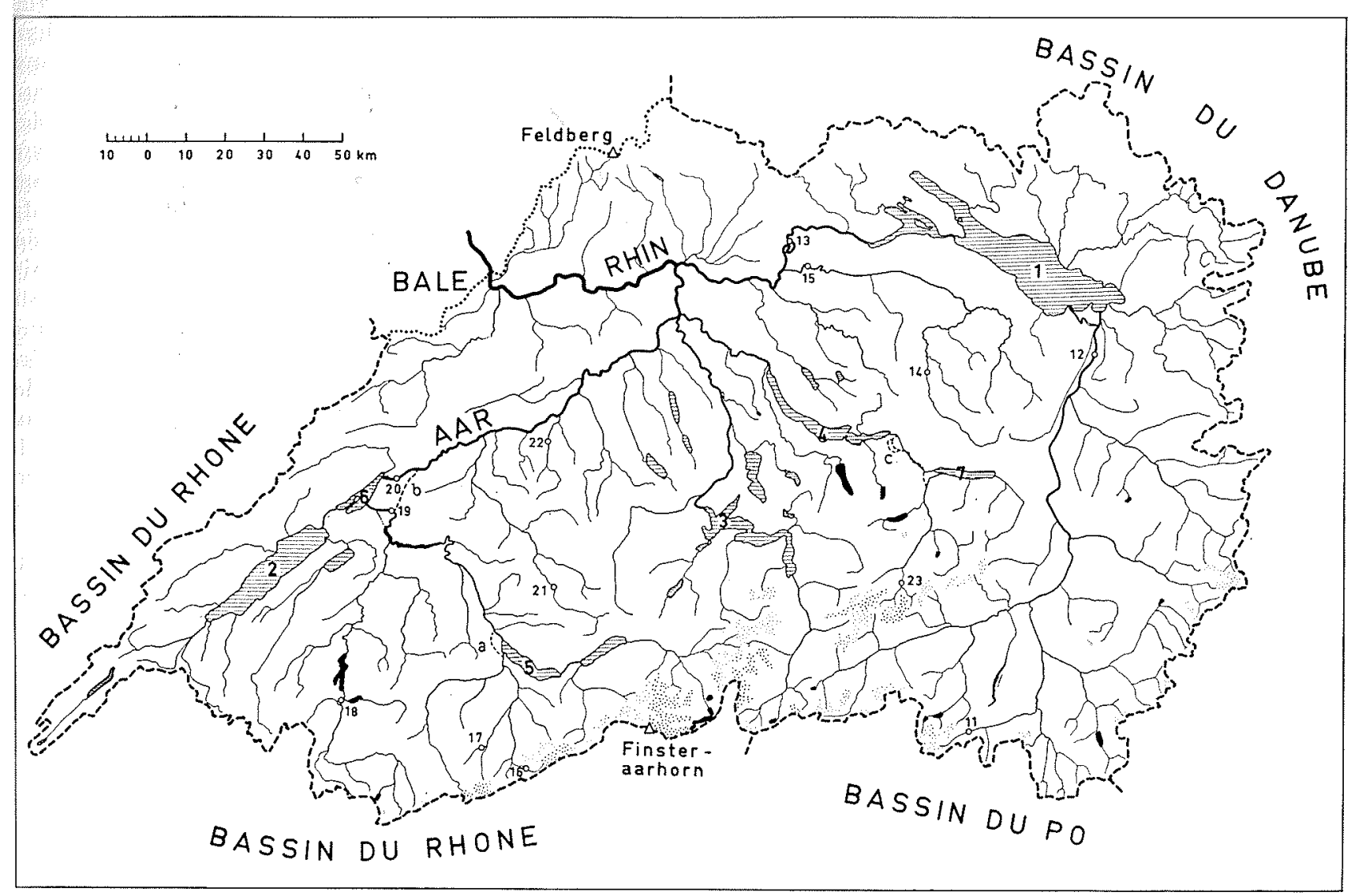

FIG. 3

Bassin du Rhin en amont de Bâle. Laes: 1. Lac de Constance, 2. Lac de Neuchâtel,

3. Lae des Quatre-Cantons, 4. Lac de Zürich, 5. Lac de Thoune, 6. Lac de Bienne,

7. Lac de Walenstadt. Ancien lit avant la correction : $a$ ) de la Kander, $b$ ) de l'Aar,

c) de la Linth. Stations: 11. Hinterrhein, 12. Schmitter, 13. Nohl, 14. Bütschwil,

15. Andelfingen, 16. Gasterntal, 17. Adelboden, 18. Broc, 19. Aarberg, 20. Brügg, 21. Eggiwil, 22. Lotzwil, 23. Tierfehd. 


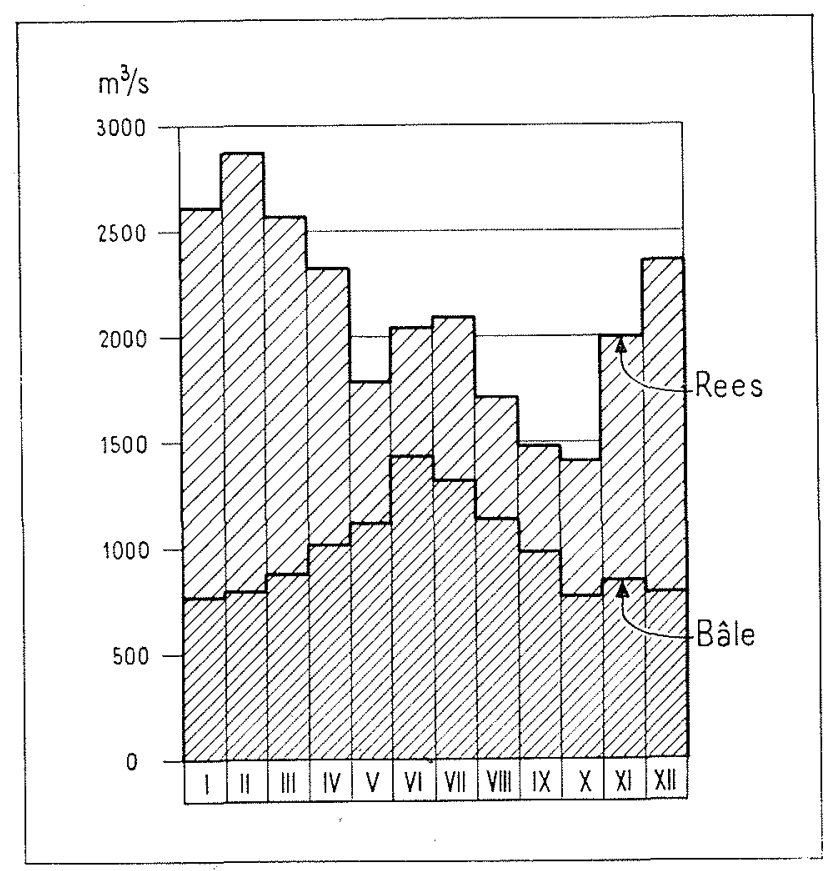

Fra. 4

Débits moyens mensuels du Rhin à Bâle et à Rees.

évidence dans le chapitre 2 du présent exposé, et nous verrons que l'altitude topographique représente un facteur déterminant pour le régime d'écoulement dans une région comportant de telles différences de niveau. 'Toutefois, nous ferons d'abord une comparaison entre quelques données relatives au régime d'écoulement du Rhin à Bâle et du même régime à Rees; pour ce faire, nous considérerons la période entre les années 1941 et 1955 , période pour laquelle nous disposions de toutes les données nécessaires [3], [4].

La valeur moyenne du débit d'écoulement était, pour la période considérée : à Bâle, $997 \mathrm{~m}^{3} / \mathrm{s}$, soit $27,3 \mathrm{I} / \mathrm{s} . \mathrm{km}^{2}$, et à Rees, $2102 \mathrm{~m}^{3} / \mathrm{s}$, soil $13,2 \mathrm{l} / \mathrm{s} . \mathrm{km}^{2}$. Presque la moitié des eaux totales du Rhin, en moyenne, s'écoulent donc déjà à Bâle, bien qu'en cet endroit la superficie du bassin de réception ne soit que d'environ le quart de celle du bassin total. La répartition sai- sonnière ressort de la figure 4 , dans laquelle nous avons représenté la moyenne des débits mensuels pour les années considérées. La valeur mensuelle maximum à Bâle se produit au mois de juin, el le minimum en janvier; Rees, par contre, atteint son maximum en février et son minimum en octobre. Le quotient des valeurs movennes mensuelles maximum et minimum est de 1,86 pour Bâle, et de 2,04 pour Rees, alor's que celui pour le bassin de réception à l'aval de Bâle à lui seul est de 3,27. Le débit maximum de courte durée enregistré à Bâle pour la période en question était de $3900 \mathrm{~m}^{3} / \mathrm{s}$, et le plus faible de $337 \mathrm{~m}^{3} / \mathrm{s}$, ce qui donne un quotient de 11,6. Les valeurs analogues pour Rees sont $9500 \mathrm{~m}^{3} / \mathrm{s}$ et $590 \mathrm{~m}^{3} / \mathrm{s}$, soit un quotient de 16,1. En résumant, on peut done dire que la contribution du bassin à l'amont de Bâle au débit d'écoulement total du Rhin est relativement importante et bien compensée.

Enfin, deux facteurs importants, et qui sont évidents même pour de non-spécialistes, établissent une différence très nette entre les bassins partiels à l'amont et à l'aval de Bâle: les glaciers et les lacs. Seul le bassin amont contient des glaciers. Leur superficie varie dans le temps, et c'est ainsi que les dernières décennies ont été accompagnées d'une régression prononcée des glaciers. Nous disposons de deux données confirmant cette régression, l'une datant d'avant l'année 1900 et l'autre du deuxième quart du siècle présent. D’après la plus ancienne donnée, la superficie des glaciers dans le bassin du Rhin était alors de $663 \mathrm{~km}^{2}$, alors que la plus récente donne le chiffre de $548 \mathrm{~km}^{2}$; ces deux valeurs correspondent d'une part à $1,82 \%$, et d'autre part à $1,50 \%$ du bassin de réception à Bâle. Le lac de Constance est le plus grand lac dans le bassin du Rhin, ayant une superficie de $542 \mathrm{~km}^{2}$, si l'on tient compte du Lac Inférieur (Untersee); suivent le lac de Neuchâtel avec $217 \mathrm{~km}^{2}$, le lac des Quatre-Cantons avec $114 \mathrm{~km}^{2}$, et le lac de $Z$ urich avec $90 \mathrm{~km}^{2}$. La superficie totale des seize plus grands lacs dans le bassin à l'amont de Bâle est de $1219 \mathrm{~km}^{2}$, c'est-à-dire $3,34 \%$ de la superficie du bassin de réception à l'amont de cette ville (fig. 3 ).

\section{II. - PRECIPITATION ET ÉCOULEMENT DANS LE BASSIN DE RÉCEPTION DU RHIN A L'AMONT DE BALE}

Entre les années 1901 et 1940 , le débit d'écoulement moyen du Rhin à Bâle était de $1075 \mathrm{~m}^{3} / \mathrm{s}$, ce qui correspond à une hauteur d'écoulement de $944 \mathrm{~mm}$ pour la région considérée. Etant donné que la hauteur des précipitations correspondantes atteignit $1420 \mathrm{~mm}$, on en déduit un coefficient d'écoulement de $66,5 \%$ (altitude moyenne du bassin : $1050 \mathrm{~m}$ au-dessus du niveau de la mer). Les contributions apportées à ces valeurs moyennes par les diverses zones d'altitude ne sont pas toutes les mêmes; les valeurs annuelles moyennes des précipitations et des dé- 
lilts d'écoulement dans les hautes régions sont plus élevées que dans les basses zones; c'est innsi qu'on a constaté une précipitation anmuelle moyenne de $1720 \mathrm{~mm}$ et un débit d'écoulement minuel moyen de $1441 \mathrm{~mm}$ (coefficient d'écouwonent de $84 \%$ ) pour un bassin partiel de caraclire alpin de $4437 \mathrm{~km}^{2}$, situé à une altitude noyenne de $2060 \mathrm{~m}$, et dont $10 \%$ de la superficie wolale est recouverte de glaciers; par contre, un louxième bassin partiel, de basse altitude et I/une superficie de $17090 \mathrm{~km}^{2}$, situé en moyenne i $630 \mathrm{~m}$ au-dessus de la mer, donnait une prévipitation annuelle moyenne de $1110 \mathrm{~mm}$ et une hauteur d'écoulement annuelle moyenne de $784 \mathrm{~mm}$, soit un coefficient d'écoulement de $63 \% \quad[3],[5],[6],[7]$

En ce qui concerne la répartition saisonnière los débits, la figure 4 fait apparaître pour Bâle 16 régime qui est souvent appelé « régime aljin $\gg$. En effet, il est caractérisé par d'imporlants débits en été et des débits plus faibles en hiver. Les considérations qui suivent ont pour wbjet de mettre en évidence quels sont les facleurs principaux participant à l'établissement de co régime.

La figure 5 représente la répartition saisonnière des précipitations, les stations pluvioméliquues correspondantes étant groupées d'après leur altitude. On voit que le régime des précipilations est sensiblement le même pour toutes les ultitudes, c'est-à-dire une abondance de précipilations en été, avec une diminution en hiver. Guant au régime des températures, on constate que l'isotherme de $0^{\circ}$ est située à une altitude denviron $3000 \mathrm{~m}$ au mois de juin. D'après la ligure 2, moins de $1 \%$ de la superficie du bassin se situe au-dessus de $3000 \mathrm{~m}$, de sorte que Ion peut dire que les précipitations estivales abondantes tombent sous forme liquide sur plus de $95 \%$ de la superficie du bassin, alimentant ainsi les cours d'eau de manière relativement directe.

Toutefois, il serait dangereux de tirer des conuusions quantitatives directes de ce qui précède. Lo pourcentage des eaux d'écoulement par rapport aux eaux de précipitation, c'est-à-dire le coefficient d'écoulement, ne varie pas seulement suivant l'altitude du terrain, comme nous venons de le voir, mais encore en fonction de la saison. Si, pour chaque zone d'altitude, la valeur du coefficient d'écoulement était indépendante du lemps, les débits estivaux dus seulement aux précipitations et sous la condition de ne tenir compte que des précipitations liquides, seraient presque de l'ordre du double des débits hivernaux. Or, ceci n'est pas tout à fait vrai, car dans de grandes parties de la région, le coefficient d'écoulement estival est plus petit qu'en hiver. Un calcul par mois du coefficient d'écoulement pour la partie la plus basse du bassin a

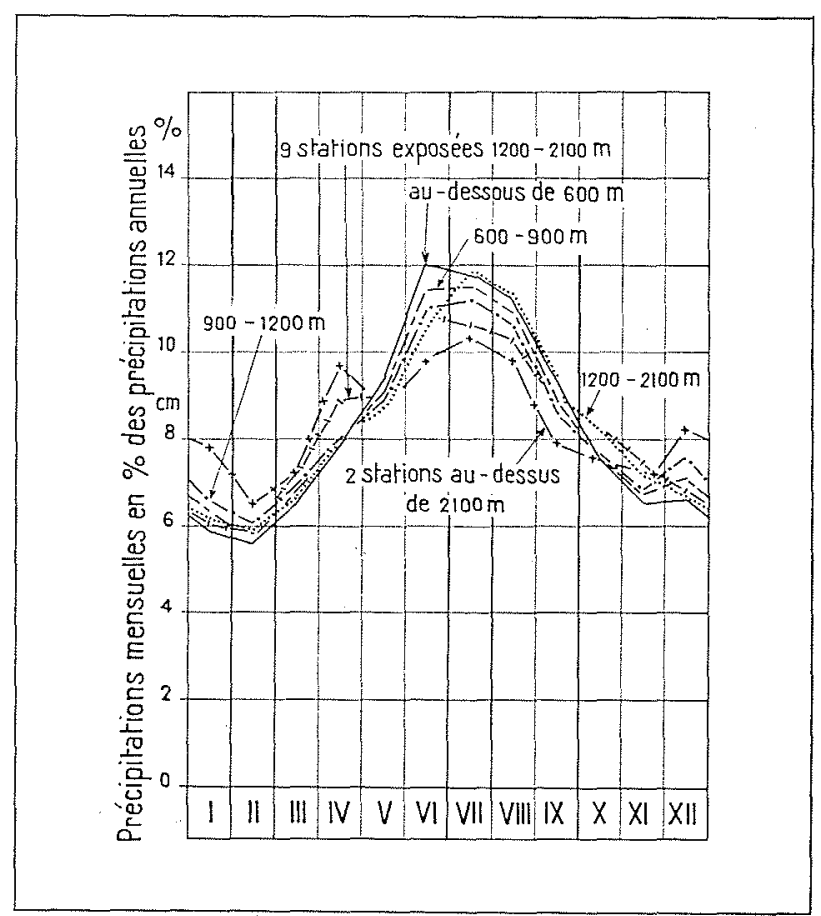

Fic. 5

Bassin du Rhin en amont de Bâle. Précipitations moyennes mensuclles en pour cent des précipitations annuelles moyennes de la période 1901-1940.

fourni des valeurs dépassant $70 \%$ pour les mois de l'hiver; pour les mois d'été, par contre, on a obtenu des valeurs inférieures à $40 \%$ [7]. Cette différence est toutefois moins grande dans les régions plus élevées, et on peut dire qu'en géné$\mathrm{ral}$, les variations saisonnières $d u$ coefficient d'écoulement diminuent la prépondérance des débits d'écoulement estivaux provoqués par l'influence directe des précipitations, mais qu'elles n'arrivent pas à l'annuler.

En hiver, une forte partie des précipitations tombe sous forme de neige, ce qui fait que l'alimentation des cours d'eau ne se produit pas immédiatement. Toutefois, la notion simpliste suivant laquelle ces précipitations « s'accumulent» pendant les mois d'hiver, pour aller ensuite s'couler « en bloc» au moment de la fonte des neiges, n'est pas tout à fait exacte. Examinons la figure 6, qui a pu être dessinée grâce à des brochures relatives à la partie autrichienne du bassin [8], mais qui peut cependant bien servir sans modifications trop importantes pour toutes les régions alpines et préalpines du Rhin. Nous voyons tout d'abord que l'on ne peut guère parler d'une couche de neige hivernale durable sur toute hauteur inférieure à $500 \mathrm{~m}$; or, d'après la figure 2, celles-ci correspondent à environ un quart du bassin de réception à l'amont de Bâle. Dans la plupart des cas, des fontes fréquentes 
noduits dans l'Emme à Eggiwil (de 1,2 $\mathrm{m}^{3} / \mathrm{s} \dot{a}$ $90 \mathrm{~m}^{3} / \mathrm{s}$ environ), et dans la Thur à Andelfingen $1.1618 \mathrm{~mm} / \mathrm{s}$ à $630 \mathrm{~mm} / \mathrm{s}$ ). Le 10 janvier, on avait whegistré une valeur moyenne journalière pour 1. Rhin à Bâle de $736 \mathrm{~m}^{3} / \mathrm{s}$, mais le 14 janvier, mu contre, le fleuve a atteint la valeur maxiinum de $3230 \mathrm{~m}^{3} / \mathrm{s}$. Ce « gonflement » du débit 1) lant persisté que l'année 1955 a finalement tumni la plus grande valeur moyenne pour le mois de janvier de toutes les valeurs enregistrées pour ce mois pendant 150 années d'observations. toutefois, on rencontre plus souvent les crues vilivales qui se produisent lorsque les crues de courte durée provoquées entre les mois de mai st juin par des précipitations importantes se suwrovosent aux débits d'écoulement de base dus ir la fonte des neiges.

les stations dont le bassin de réception conlinnt une forte part de glaciers se prêtent parti- culièrement bien à l'observation du régime purement alpin, comme le met en évidence la figure 7 pour la Linth à Tierfehd [3]. Ici, des crues hivernales ne se produisent plus; en revanche, pendant les mois d'été, les débits ne descendent jamais au-dessous d'une certaine valeur, relativement élcvée. En ce qui concerne la Linth, on voit d'après la figure 7 que les valeurs instantanées les plus grandes que l'on ait jamais observées pour la période janvier/mars, étaient néanmoins toujours inférieures aux plus petites moyennes journalières enregistrées pour les mois de juillet et août. La totalité des moyennes men. suelles enregistrées pour le mois de février se situe entre 0,21 et $0,72 \mathrm{~m}^{3} / \mathrm{s}$, et celle du mois de juin entre 6,66 et $19,7 \mathrm{~m}^{3} / \mathrm{s}$. La différence entre les débits d'été et d'hiver est très grande, comme on peut le voir d'après le tableau ci-dessous :

\begin{tabular}{|c|c|c|c|c|}
\hline \multirow{2}{*}{ Cours d'eau } & \multirow{2}{*}{ Station } & \multicolumn{2}{|c|}{ Bassin de réception } & \multirow{2}{*}{$\begin{array}{c}\text { Quotient des débits moyens de juin } \\
\text { ou juillet, et de février }\end{array}$} \\
\hline & & Altitude moyenne & Glaciation en $\%$ & \\
\hline Ironder ..... & Gasterntal ... & $2600 \mathrm{~m}$ & $43,5 \%$ & 49 \\
\hline 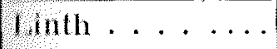 & Tierfehd...... & $2330 \mathrm{~m}$ & $23,9 \%$ & 33 \\
\hline $\begin{array}{r}\text { Hinterthein (Rhin } \\
\text { postérieur) }\end{array}$ & Hinterrhein & $2390 \mathrm{~m}$ & $21,0 \%$ & 26 \\
\hline
\end{tabular}

On sait qu'une partie de ces grands débits esIivunx est retenue dans des lacs d'accumulation whificiels; cette partie, lorsqu'elle est exploitée purr la production d'énergie hydro-électrique hivornale, contribue à l'augmentation du débit hivernal normalement faible des parties aval des cours d'eau. L'autre partie augmente directemont le débit estival, surtout pendant les mois 1. juillet et août, car c'est à cette époque de Tannée que les lacs naturels ont généralement ulja dépassé leur cote maximum; non seulemont ne retiennent-ils alors plus d'eau, mais ils coutribuent encore par des apports d'eau à l'acroissement du débit de leurs cours d'eau exucotres.

L'effet combiné des différentes composantes produit finalement le régime d'écoulement que nous pouvons observer à Bâle. L'observation systématique des niveaux du Rhin à Bâle remonte à l'année 1808; la détermination des débits en cet endroit date également de très loin, et, sur la base de certaines études [9], les débits mensuels moyens furent reconstitués pour les années à partir desquelles furent faites les premicres observations, de sorte que nous disposons actuellement d'une vue d'ensemble du régime d'écoulement du Rhin à Bâle pour une période de 150 ans. C'est grâce à cela qu'il a été possible d'étudier les variations des débits d'écoulement annuels, en utilisant les méthodes de la statistique mathématique moderne pour la recherche des oscillations périodiques d'une durée de plusicurs années $[10]$.

\section{III. - INFLUENCE DES LACS}

Le rôle important des lacs dans le régime saisonnier des débits a déjà été évoqué dans les chapitres précédents. Entre les mois de mars et do juin, ces lacs retiennent une partie des eaux qui les alimentent, les restituant ensuite en tant que complément à partir du mois de juillet jusqu'au mois de février suivant. Signalons, à titre d'exemple, que d'après les calculs effectués, le débit d'écoulement moyen, pendant le mois de imin. du Rhin à Bâle, pendant les années 1921 
se produisent même au fort de l'hiver sur ces hauteurs, de sorte que la neige n'y subsiste tout au plus que pendant quelques semaines. En général, la durée de l'enneigement est d'autant plus longue que la région en question est haute. Ceci se voit très nettement sur la figure 6: de plus, les courbes pour févier-mai montrent clairement comment les températures croissantes au printemps, lorsque les isothermes « grimpent », repoussent, avec le temps, la zone des fontes de neige intenses de plus en plus vers les grandes hauteurs. Ce phénomène est également mis en évidence par les mesures de débit effectuées aux diverses stations dont les bassins de réception non glaciaires se trouvent à des altitudes différentes (valeur moyenne de levés s'étendant sur plusieurs années).

L'influence de la fonte des neiges sur les débits d'écoulement du Rhin à Bâle est encore diminuée par le fait que de grandes parties du bassin se trouvent à l'amont des lacs et que ces

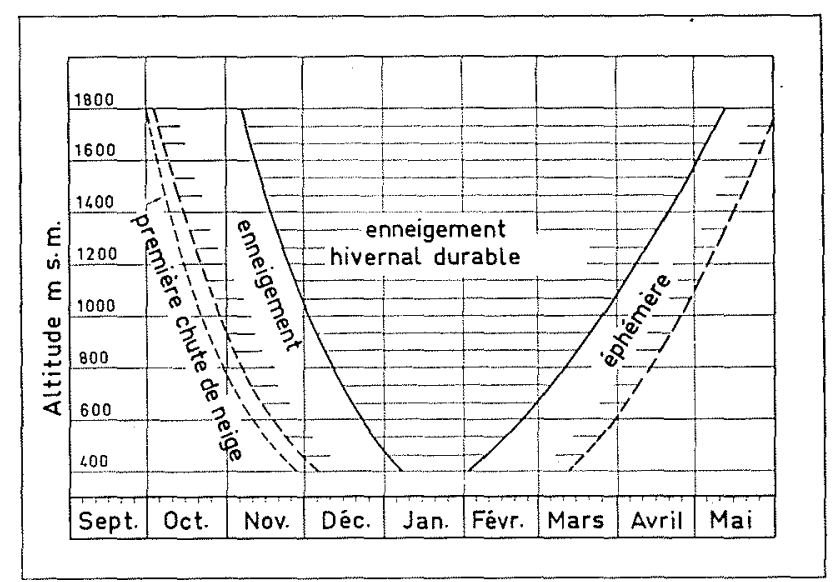

Fri. 6

Bassin autrichien du Rhin : Durée moyenne de l'enneicement en fonction de l'altitude. Début et fin de l'enneigement éphémère et de l'enneigement durable, ainsi que l'apparition de la première neige en fonction de l'altitude. Moyennes de 20 i 50 ans pour 17 stations.

\begin{tabular}{|c|c|c|c|}
\hline Cours d'eau & Station & $\begin{array}{l}\text { Altitude moyenne } \\
\text { du bassin de } \\
\text { réception. }\end{array}$ & $\begin{array}{l}\text { Mois pendant lequel se produit } \\
\text { le plus important } \\
\text { des } 12 \text { débits d'écoulement mensuels. }\end{array}$ \\
\hline Langeten . . & Lotzwil . . . . . & $740 \mathrm{~m}$ & Mars \\
\hline Thur & Bütschwil . . . . & $1110 \mathrm{~m}$ & Avril \\
\hline Emme ... & Eggiwil .... & $1330 \mathrm{~m}$ & Avril \\
\hline Allenbach & Adelboden .... & $1850 \mathrm{~m}$ & Mai \\
\hline
\end{tabular}

derniers atteignent leur niveau minimum annuel autour de fin février/début mars. Une grande partie des eaux de fonte provoque le remplissage des lacs pendant les mois de mars à juin, cette eau ne parvenant ensuite que plus tard au système fluvial situé plus bas. Nous reparlerons de l'effet compensateur des lacs dans le troisième chapitre.

Par ailleur's, les fontes hivernales ne sont pas toujours limitées à la zone la plus basse. Il peut exceptionnellement se produire que, même en janvier, l'isotherme des $0^{\circ}$ monte vers les $2000 \mathrm{~m}$. Ces irruptions d'air chaud proviennent de l'ouest et s'accompagnent souvent de pluies abondantes. Ceci provoque également la fonte de quantités appréciables de neige sur des hauteurs, même au-dessus de $1500 \mathrm{~m}$, ce qui peut entraîner des crues hivernales. Pendant la période du 10 au 13 janvier 1955 par exemple, un de ces brusques changements du temps a provoqué une crue de la Sarine à Broc, pendant laquelle le débit est passé de $9,2 \mathrm{~m}^{3} / \mathrm{s}$ à la valeur maximumi instantanée de $330 \mathrm{~m}^{3} / \mathrm{s}$ environ; par ailleurs, des accroissements analogues se sont

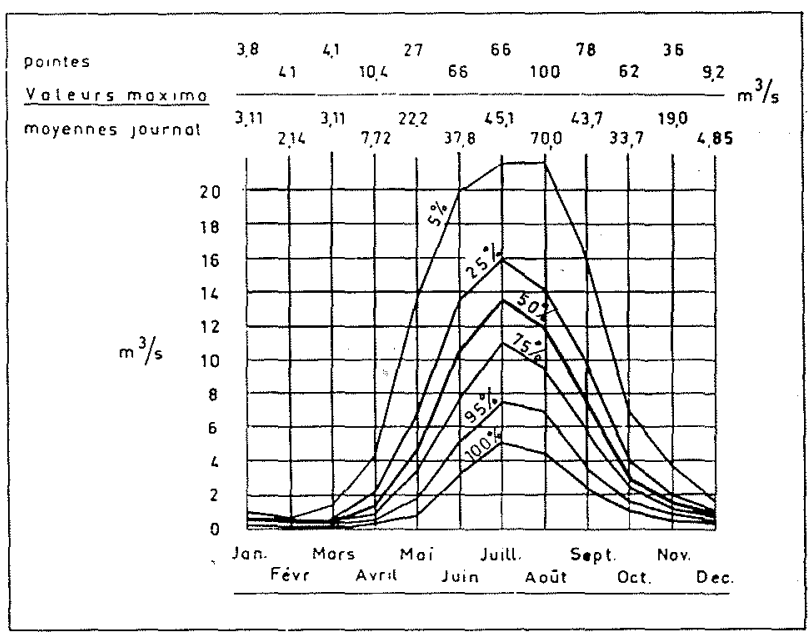

Fig. 7

Durée mensuelle moyenne des débits de la Linth à Tierfehd pour la période 1918-1957. Glaciation 23,9\%. Un point de la ligne brisée $25 \%$ indique, par exemple, que dans le mois considéré le débit a atteint ou dépassé pendant $25 \%$ des jours la valeur correspondante lue sur l'ordonnée. 


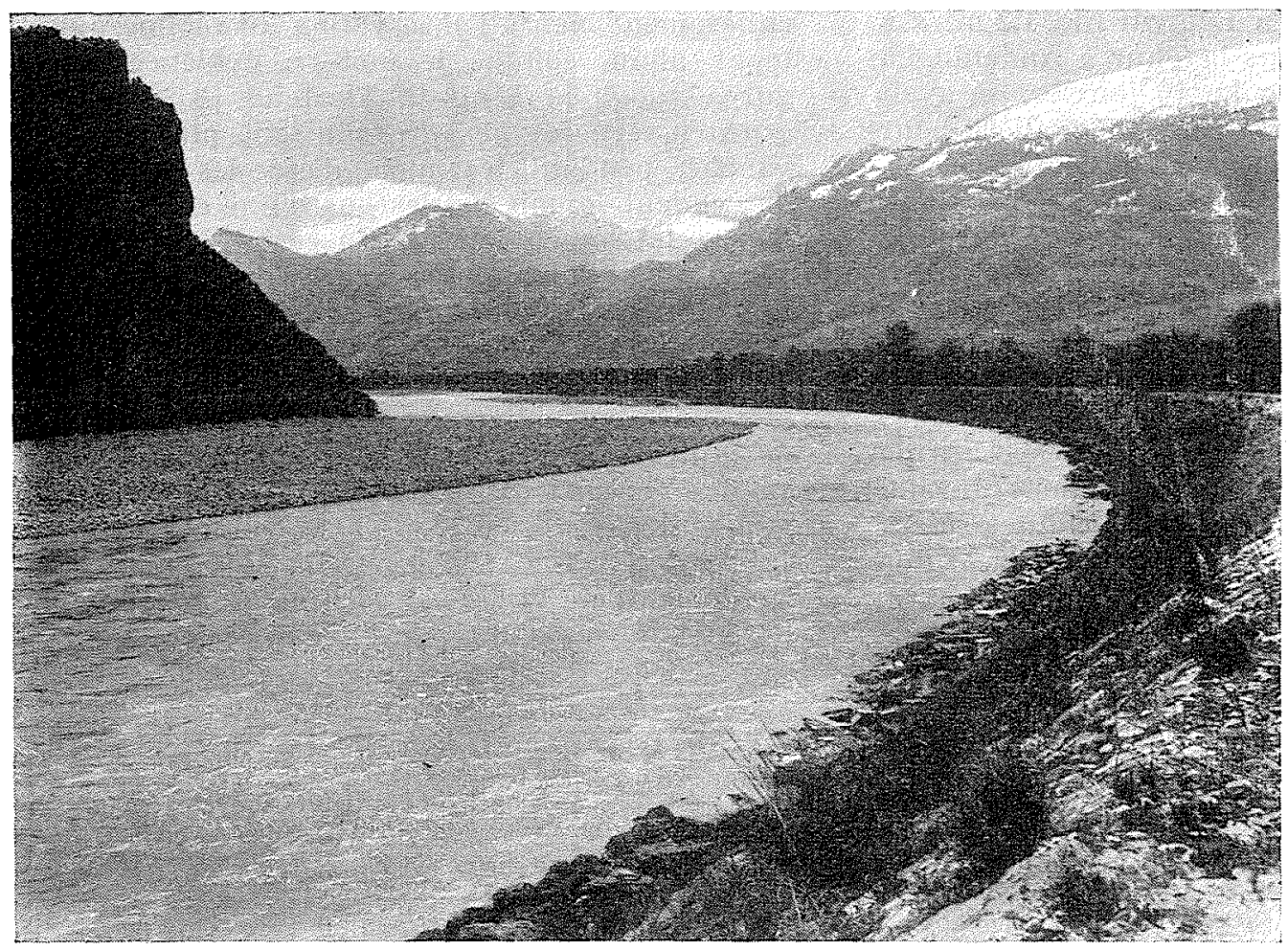

FiG. 8

Rhin en amont $\mathrm{du}$

late de Constance. Digues, banc's de gravier. à 1952 , aurait été de $96 \mathrm{~m} 3 / \mathrm{s}$ plus grand, s'il n'y avait pas en de lacs naturels. Réciproquement, la libération de quantités d'eau par les lacs naturels a augmenté le débit du Rhin, pendant le mois de janvier, d'un complément moyen de $37 \mathrm{~m}: \mathrm{s}$. Quant à l'influence des lacs d'accumulation, leur apport estimatif de janvier à Bâle se situe actuellement autour de $60 \mathrm{~m}^{3} / \mathrm{s}[7]$.

Si nous choisissons parmi les débits d'écoulement moyens mensuels d'une période assez longue les valeurs maximum et minimum, et si nous en constituons le quotient, nous obtenons, pour la station de Schmitter, à l'amont du lac de Constance: $520 \mathrm{~mm} / \mathrm{s}$ (juin); $72,4 \mathrm{~m}^{3} / \mathrm{s}$ (février) $=7,2$, et pour la station de Nohl, à l'aval du même lac : $635 \mathrm{~m}^{3} / \mathrm{s}$ (juillet); $205 \mathrm{~m}^{3} / \mathrm{s}$ (février) $=3,1$. A cet égard, l'effet compensateur des lacs sur les phénomènes d'écoulement est d'autant plus prononcé que ces derniers sont de courte durée. Si nous répétons le calcul que nous venons de faire, mais cette fois en faisant intervenir, au lieu des valeurs mensuelles, la valeur instantanée maximum et la plus petite moyenne journalière de la période, nous obtenons alors un quotient de 65 pour la station en amont du lac de Constance, et de 10,3 pour celle à l'aval. Le débit d'écoulement maximum du Rhin avant son embouchure dans le lac de Constance, où le bassin de réception s'étend sur $6122 \mathrm{~km}^{2}$, était de $2300 \mathrm{~m}^{3} / \mathrm{s}$ lors de la crue de septembre
1927; par contre, à l'aval du lac, bien que la superficie du bassin de réception en ce point soit presque le double de celle en amont, le débit d'écoulement n'est monté que jusqu'à $900 \mathrm{~m}^{3} / \mathrm{s}$ à la même époque. Par ailleurs, on a calculé pour le seul bassin de réception du lac de Zürich $\left(768 \mathrm{~km}^{2}\right)$, que lors de la crue de juin 1953, environ le quart des précipitations de 150 millions de $\mathrm{m}^{3}$ enregistrées pour les quatre jours décisifs a été retenu par le sol, et que par contre, environ la moitié a été retenue par le lac, de sorte qu'un quart seulement est parvenu directement au Rhin pendant cette période.

Les limites des variations du plan d'eau, et la rapidité et la fréquence des intumescences, ajoutées à d'autres facteurs, influent considérablement sur le caractère du paysage le long du fleuve en question. Les différences entre deux troncons du Rhin, l'un en amont du lac de Constance, et l'autre à l'aval, se voient très nettement sur les figures 8 et 9 . Il convient également à cet égard de signaler le rôle des lacs en tant que collecteurs de matériaux solides. L'aspect de la région à l'aval du lac de Constance serait en effet très différent, si les débits de crue pouvaient s'écouler sans obstacle de l'amont vers l'aval, et si les quelque $3,5 \mathrm{mil}$ lions de $\mathrm{m}^{3}$ de matériaux solides déposés annuellement dans le lac étaient charriés par te troncon aval du fleuve [11].

Par ailleurs, les lacs compensent également 
FI(i. 9

Rhin en aval du

lac de Constance. Faibles variations du niveau de l'ean.

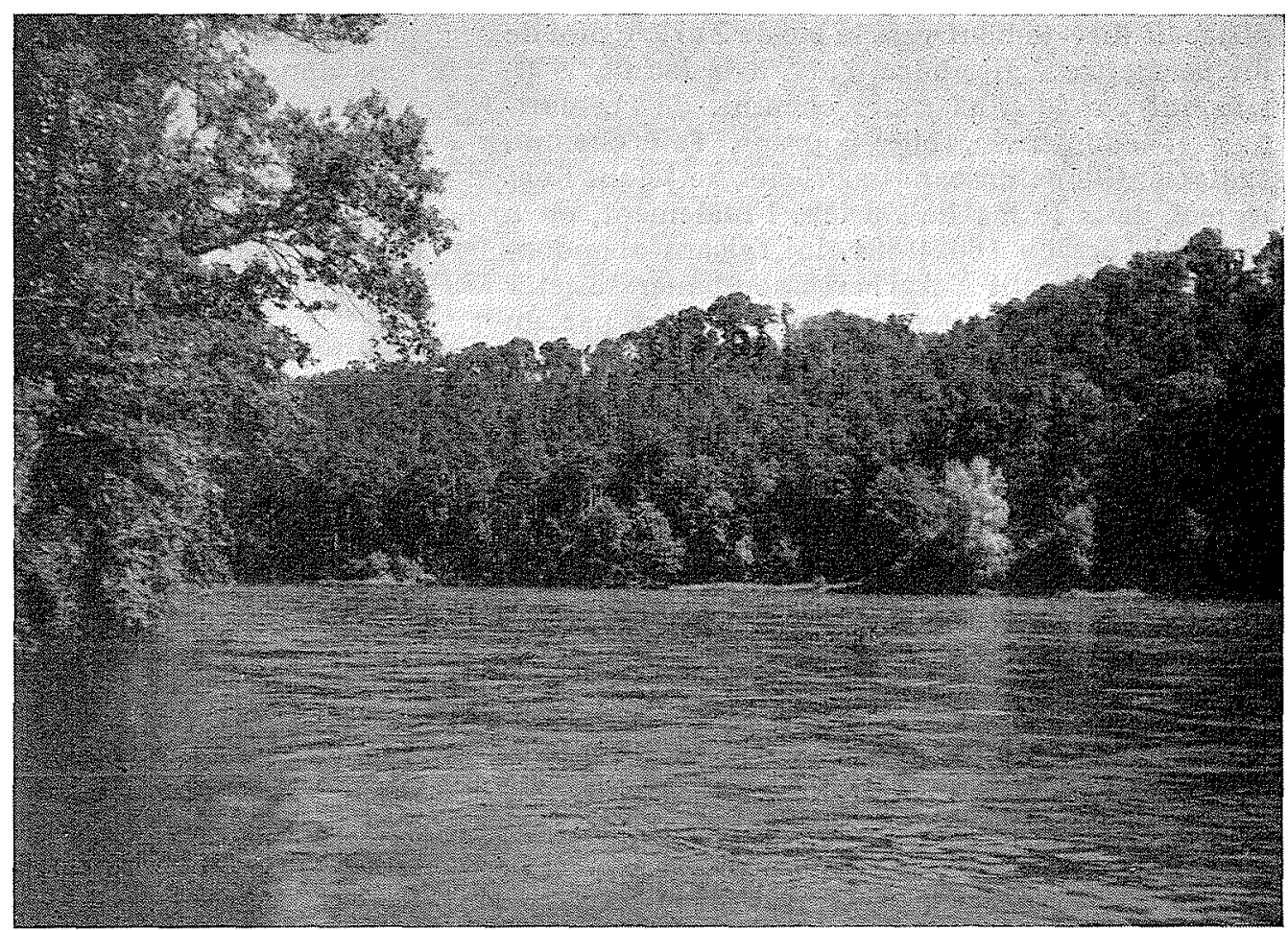

les variations de débit de courte durée pouvant être provoquées par le fonctionnement des usines hydro-électriques [12] (fig. 10).

Il ressort de ce qui précède que la manière selon laquelle se font les apports d'eau dans le cours supérieur d'un fleuve, soit par écoulement direct, soit par l'intermédiaire de lacs, est d'une importance primordiale pour le régime d'écoulement de ce fleuve. Pour le Rhin à Bâle, nous avons calculé la grandeur de la partie du bassin de réception fournissant des apports d'eau directs par écoulement, sans compensalion par des lacs, en déterminant les bassins de réception correspondant aux premiers lacs que l'on rencontre en remontant le fleuve et ses divers confluents à partir de Bâle, et en les soustrayant de la superficie du bassin de réception global. Ce faisant, il nous a fallu tenir compte du fait que différentes rivières importantes, qui se jetaient autrefois librement dans le Rhin, ont cté déviées de leur lit et dirigées dans des lacs par les travaux de correction effectués pendant ces derniers siècles. L'objet de ces travaux était de libérer les parties aval de ces rivières du transport de matériaux solides, afin de créer les conditions nécessaires à la réalisation d'une protection permanente et efficace contre les crues. La plus ancienne de ces mesures que nous connaissions est la dérivation de la Kander réalisée de 1711 à 1714 , qui faisait débiter cette rivière dans le lac de Thoune au lieu de se jeter dans l'Aare à l'aval de Thoune comme elle le faisait auparavant (fig. 3). Par ailleurs, pendant les années 1807-1827, on a creusé un nouveau lit pour la Linth, pour remplacer l'ancien lit qui se

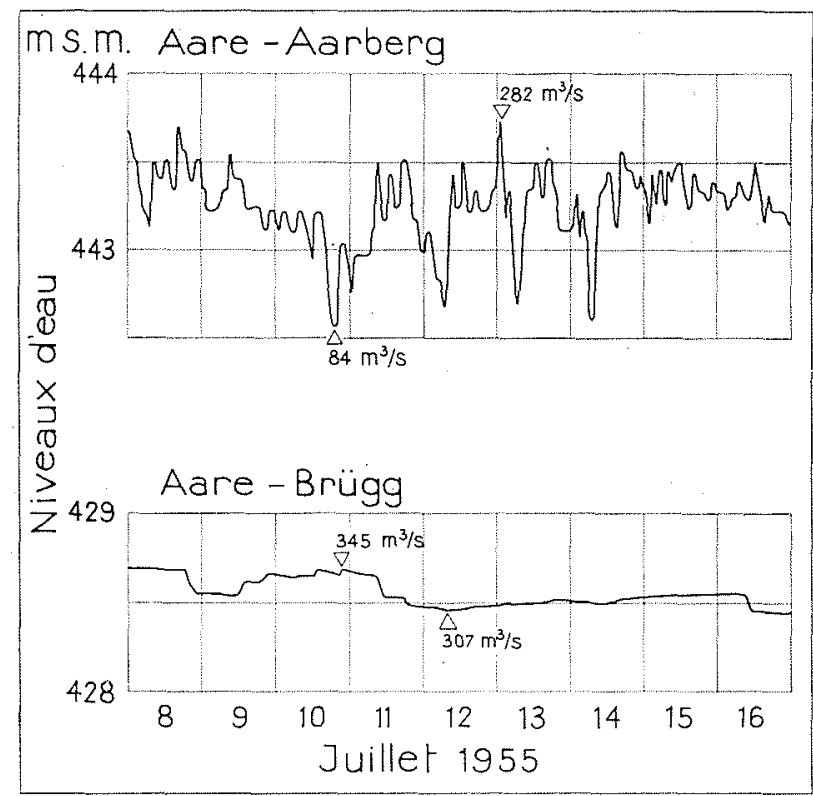

F.IG. 10

Effet régulateur du lac de Bienne. Débit de l'Aare à Aarberg (influencé par des usines) et à Brügg (en aval du lac de Bienne régularisé). Le 10 juillet: dimanche. 
dirigeait directement vers le lac de Zurich, afin de lui permettre d'atteindre d'abord le lac de Wallenstadt par le plus court chemin; de là, clarifiée et compensée, elle poursuit son chemin, également dans un canal artificiel, se jetant finalement dans le lac de Zürich. Finalement, on a dérivé l'Aare en 1869-1891, lui faisant abandonner son ancien lit à Aarberg, pour aller se jeter dans le lac de Bienne, d'où elle ressort par un lit artificiel et rejoint son ancien lit $12 \mathrm{~km}$ à l'aval de la dérivation.

Si nous calculons la superficie du bassin fournissant les apports d'eau au Rhin en amont de Bâle avant l'année 1711 sans traverser un des grands lacs, nous obtenons un chiffre de $15797 \mathrm{~km}^{2}$. L'aménagement de la Kander a réduit cette superficie de $1352 \mathrm{~km}^{2}$, puis est survenue une deuxième réduction lors de l'aménagement de l'Aare, cette fois de $2436 \mathrm{~km}^{2}$. Si l'on tient compte encore de quelques autres aménagements d'ordre mineur, on peut aussi dire, réciproquement, que la superficie des bassins de réception compensée par des lacs, de $20695 \mathrm{~km}^{2}$ à l'origine, s'est trouvée augmentée de $19 \%$ par des moyens artificiels, de sorte qu'elle est actuellement de $24639 \mathrm{~km}^{2}$ soit $67,5 \%$ du bassin de réception total jusqu'à Bâle. (Nous n'avons pas tenu compte des bassins d'accumulation alpins dans ce calcul.) La tendance régressive actuelle du quotient du débit maximum d'écoulement instantané par la valeur moyenne journalière minimum du Rhin à Bâle est sans doute liée, du moins en partie, aux aménagements que nous venons d'exposer. Cette tendance est mise en évidence dans le tableau ci-dessous :

Période Débitmax. (année): Débit min. (année) $=$ Quotient

$\begin{array}{llllll}1908 \text { à } 1900 . & 5700 & (1876): & 202 & (1858)= & 28,2 \\ 1901 \text { à } 1920 . & 4300 & (1910): & 272 & (1909)= & 15,8 \\ 1921 \text { à } 1957 . & 3800 & (1953): & 306 & (1921)= & 12,4\end{array}$

Ceci n'exclut aucunement la possibilité d'une répétition ou un dépassement de la crue maximum de $3800 \mathrm{~m}^{3} / \mathrm{s}$ dans l'avenir; mais par contre, il est invraisemblable que les débits minima, dont la valeur a été accrue surtout par l'influence des lacs d'accumulation, retrouvent leurs valeurs d'antan.

\section{BIBLIOGRAPHIE}

[1] Les Forces hydrauliques de la Suisse, A. Les surfaces des bassins de réception, Berne, 1916.

[2] Régime des eaux en Suisse. Surfaces des bassins de réception, des zones de 300 en $300 \mathrm{~m}$ au-dessus de la mer, des rochers, des forêts, des glaciels et des lacs, Berne, 1896 (Bassin du Rhin), 1903 (Bassin de la Reuss), 1910 et 1920 (Bassin de l'Aar), 1920 (Bassin de la Limmat).

[3] Service fédéral des eaux: Annuaire hydrographique de la Suisse, paraît annuellement à Berne.

[4] Deutsches Gewässerkundliches Jahrbuch, Niederrheingebiet, parait annuellement at Düsseldorf.

[5] Institut Suisse de Métérologie : Ergebnisse der täglichen Niederschlagsmessungen, paraît annuelment à Zürich.

[6] Uttinger, Institut Suisse de Météorologie: Les plécipitations en Suisse 1901-1940, Zürich, 1949.

[7] Walser : Die Niederschlags- und Abflussverhältnissc im Einzugsgebiet des Rheins oberhalb Basel. «Cours d'eau et énergie $", \mathrm{~N}^{\circ} 5-7,1954$, Zürich.
8] Hydrographischer Dienst in Oesterreich, Beiträge zur Hydrographie Oesterreichs. Heft Nr. 25: Die Schneeverhältnisse in Oesterreich im Zeitraum 1901 bis 1950, Teil I. hrg. vom Hydrographisehen Zentralbureau, Wien, 1952.

[9] Communication $\mathrm{n}^{\circ} 19$, du Service fédéral des eaux : Ghezzi : Die Abflussverhältnisse des Rheins in Basel, Berne, 1926.

[10) Communication no 38 du Service fédéral des eaux: Oesterhaus: Oscillations périodiques d'une duré de plusieurs années du débit du Rhin à Bâle, Berne, 1946.

11) Communication n" 34 du Service fédéral des eaux Deltaaufnahmen des Eidg. Amtes für Wasserwirt. schaft, Berne, 1939.

12] Walser und Lanker : Niederschlags- und Abflussverhältnisse in Einzugsgebiet der Aare unte bessonderer Berücksichtigung des Einflusses der Speicherbecken. «Cours d'ean et énergie». Nos 7 . 9, 1957, Zïrich. 